\title{
Hearing Loss and Associated Factors in Iranian Patients with Type 2 Diabetes: A Case-Control Study
}

\author{
Saeed Nouri, ${ }^{1}$ Raika Jamali, ${ }^{2,3,}{ }^{*}$ Kurosh Gharagozli, ${ }^{1}$ Mohammad Reza Sharif, ${ }^{4}$ and Bardia Jamali ${ }^{5}$ \\ ${ }^{1}$ Department of Neurology, Shahid Beheshti University of Medical Sciences, Tehran, IR Iran \\ ${ }^{2}$ Research Development Center, Sina Hospital, Tehran University of Medical Sciences, Tehran, IR Iran \\ ${ }^{3}$ Anatomical Sciences Research Center, Kashan University of Medical Sciences, Kashan, IR Iran \\ ${ }^{4}$ Trauma Research Center, Kashan University of Medical Sciences, Kashan, IR Iran \\ ${ }^{5}$ Faculty of Pharmacy, Department of Pharmaceutics, Tehran University of Medical Sciences, Tehran, IR Iran \\ "Corresponding author: Raika Jamali, Research Development Center, Sina Hospital, Tehran University of Medical Sciences, Tehran, Iran. Tel: +98-2163120000, E-mail: \\ jamalira@tums.ac.ir
}

Received 2016 December 06; Revised 2016 December 10; Accepted 2016 December 10.

\begin{abstract}
Background: Hearing loss is an important impairment that occurs in the diabetics and affects their quality of life. There is still a paucity of studies on the relationship between diabetes and hearing loss.

Objectives: This case-control study compared the prevalence of hearing loss in a sample of diabetics with that in an age and sex matched group of healthy controls.

Methods: Cases were consecutively selected from the diabetics registered in two diabetes centers during February to December 2013. Controls were age and sex matched healthy volunteers admitted to a blood bank. Pure tone audiometric test (PTA) was performed by audiometer for the evaluation of hearing impairment. Then, the existence of hearing impairment in different intensities was compared between the two groups considering the effect of diabetes duration, BMI, and serum creatinine (Cr) level.

Results: Eighty diabetics and eighty healthy controls were included. Hearing loss in sounds with high frequencies was more common in the diabetics than the controls. The mean serum creatinine level was significantly higher in the diabetics with hearing loss than those without hearing loss at noise frequencies of $4 \mathrm{KHz}$ and $8 \mathrm{KHz}(\mathrm{P}<0.05)$. Meanwhile, hemoglobin A1C, duration of diabetes, and body mass index were not associated with hearing loss.

Conclusions: According to the results, hearing impairment in high frequency sounds was more common in the diabetics than the healthy individuals. Also, the severity of hearing loss in patients with diabetes was associated with the levels of creatinine (diabetic nephropathy). The hypothesis of the relationship between diabetes and hearing loss through nephropathy should be more examined in future studies
\end{abstract}

Keywords: Hearing Loss, Glycosylated Hemoglobin A, Creatinine, Glycosylated Hemoglobin A, Body Mass Index

\section{Background}

Diabetes mellitus is a metabolic disorder characterized by chronic hyperglycemia and impaired metabolism of carbohydrate, fat and protein (1). The prevalence of diabetes is increasing rapidly (2). Diabetic complications have great socioeconomic impacts on the public health (3-5). Hearing loss is an impairment in the diabetics that lowers their quality of life. There are controversial results regarding its association with diabetes (6-9). Age, levels of kidney function impairment, hypertension, atherosclerosis, and glycemic control are the proposed factors that could be associated with hearing loss in diabetics (6-9). It seems to be a logical approach to evaluate the relationship between hearing loss and diabetes by considering the above mentioned factors.

The aims of the study were: 1) to compare the prevalence of hearing loss among the diabetics with that among the healthy controls. 2) to define the relationship between hearing loss and serum creatinine $(\mathrm{Cr})$, hemoglobin A1c, body mass index (BMI), and the duration of diabetes.

\section{Methods}

\subsection{Patient and Control Enrolment}

This case-control study was conducted during February to December 2013. Subjects in the case group were consecutively selected from the diabetics who referred for diabetic control to Golabchi and Naghavi diabetes centers in Kashan University of Medical Sciences. The inclusion criteria for the case group were a medical diagnosis of diabetes mellitus (based on the criteria of American diabetes association 2013), being under treatment with antidiabetic medications or insulin, and an age between 20 to 50 years. The exclusion criteria were as follows: 1 ) existing excessive ear wax in the ear canal or any abnormality in the eardrum detected by otoscopy, 2) active otitis media, 3) complicated otitis media, 4) history of ear 
surgery, cerebrovascular accident, meningitis, otosclerosis, measles, mumps, and rubella, 5) history of receiving ototoxic medications (i.e. aminoglycosides), 6) history of having a job with high levels of noise pollution, 7) history of hypothyroidism (serum thyroid stimulating hormone (TSH) level more than $5(\mathrm{U} / \mathrm{mL})), 8$ ) history of head injury, 9) known causes of chronic renal failure other than diabetic nephropathy.

Subjects in the control group were selected among healthy individuals admitted to Kashan's blood bank to donate blood. A biostatistician matched the cases and the controls (block matching method) with regard to age and sex.

\subsection{Audiometric Studies}

Hearing loss was defined if there was decreased sensitivity to the sounds in the speech frequencies (8). Normal hearing threshold was set at the power of 20 decibel $(\mathrm{dB})$ in this study (8). Pure tone audiometric test (PTA) was performed by an audiometer that was capable of producing acoustic tones in adjustable frequencies. To avoid the inter-observer variability of the audiometric findings, the test was performed by an expert audiology technician. This test evaluated the patients' hearing status, diagnosed different hearing disorders, and helped the patients to use assistive hearing devices. The frequency distribution of different hearing impairments was obtained.

\subsection{Laboratory Measurements}

A blood sample was obtained from each subject after eight hours of fasting to measure the levels of HbA1c, TSH, and $\mathrm{Cr}$. The laboratory assays were performed by enzymelinked immunosorbent assay (ELISA) method (using Pars Azmoon Kit, Tehran, Iran) according to the manufacturer's instruction. All laboratory data were collected in duplicate at a standard laboratory environment regarding temperature and humidity. The inter-assay variability of the measured laboratory findings was less than $10 \%$ in this project. The checklist used for clinical data collection included data on the subjects' age, gender, height, weight, BMI, duration of diabetes, and the name of the drugs used. Also, there was a special form for recording the results of the subjects' laboratory examinations including HbA1c, TSH, Cr, glomerular filtration rate (GFR), and the result of the audiometric examinations. The study protocol was completed for all the subjects through individual interviews in a private environment at the diabetes clinics or in the blood bank.

Patients were divided into healthy weight, overweight, and obese according to their BMI.

\subsection{Ethical Considerations}

Research ethics committee of Kashan University of Medical Sciences approved the study protocol (registration number: 9155). All subjects were briefed about the study objectives before the start of the study. They all signed a written informed consent before enrolment. All subjects were also assured of the confidentiality of their personal information.

\subsection{Sample Size Calculation}

The sample size was expected to be 81 in each study group in view of mean prevalence of hearing loss in the diabetics $(\mathrm{P}=27 \%, \alpha=0.05, \mathrm{z}=1.96$, and $\mathrm{d}=0.2)$.

\subsection{Statistical Analysis}

Kolmogorov-Smirnov test was used for analyzing the normal distribution of data. Two-sample t-test was used to compare the mean values of continuous variables (age, creatinine, GFR, and HbA1c) in the diabetics with those in the healthy volunteers. Chi-square test was used to compare categorical variables of the study groups. Pvalue (twosided) less than 0.05 was considered as the significant level all through the statistical analysis. Data analysis was performed using SPSS 19 (IBM, Inc., Armonk, NY).

\section{Results}

One hundred and eighty diabetic patients were visited in the mentioned diabetic centers. The number of the excluded patients and the cause of their exclusion is shown in Figure 1.

Finally eighty diabetic patients and eighty age and sex matched healthy subjects were recruited in this study. The mean duration of diabetes in the case group was 7.92 ( \pm 5.22) years, ranging from 1 to 20 years. The patients' characteristics are shown in Table 1. The mean creatinine and GFR were not significantly different in the study groups ( $P$ $>0.05$ ). However, the mean HbA1c was significantly higher among the diabetic patients, which signifies inappropriate glycemic control (9.09 vs. 3.84, $\mathrm{P}<0.001)$. The distribution of hearing loss among the two study groups is demonstrated in Table 2. The comparison of mean serum creatinine level in the diabetic patients with and without hearing loss is provided in Table 3.

In all of the sound frequencies, no significant differences were observed between the diabetics with and without hearing loss in terms of mean diabetes duration, HbA1C, and BMI. However, the mean creatinine was significantly different in the diabetics with and without hearing loss at the sound frequencies of $4 \mathrm{KHz}$ and $8 \mathrm{KHz}$ (Table 3 ). 
Assessed for eligibility fro control group $(n=96)$

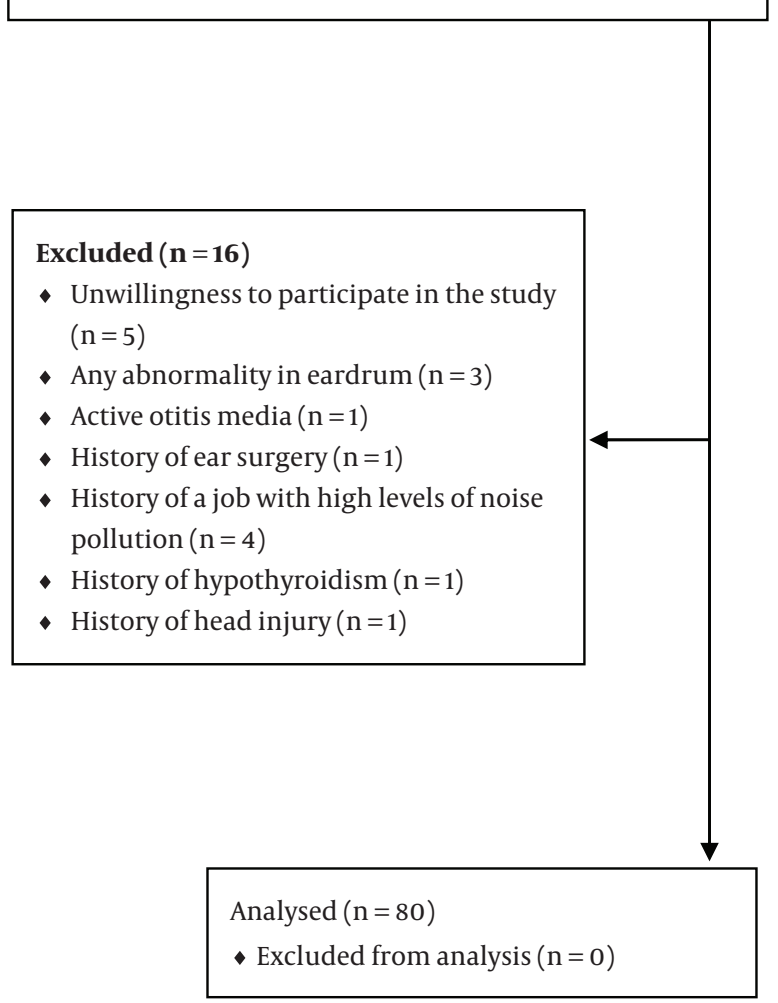

Assessed for eligibility fro case group $(\mathrm{n}=130)$

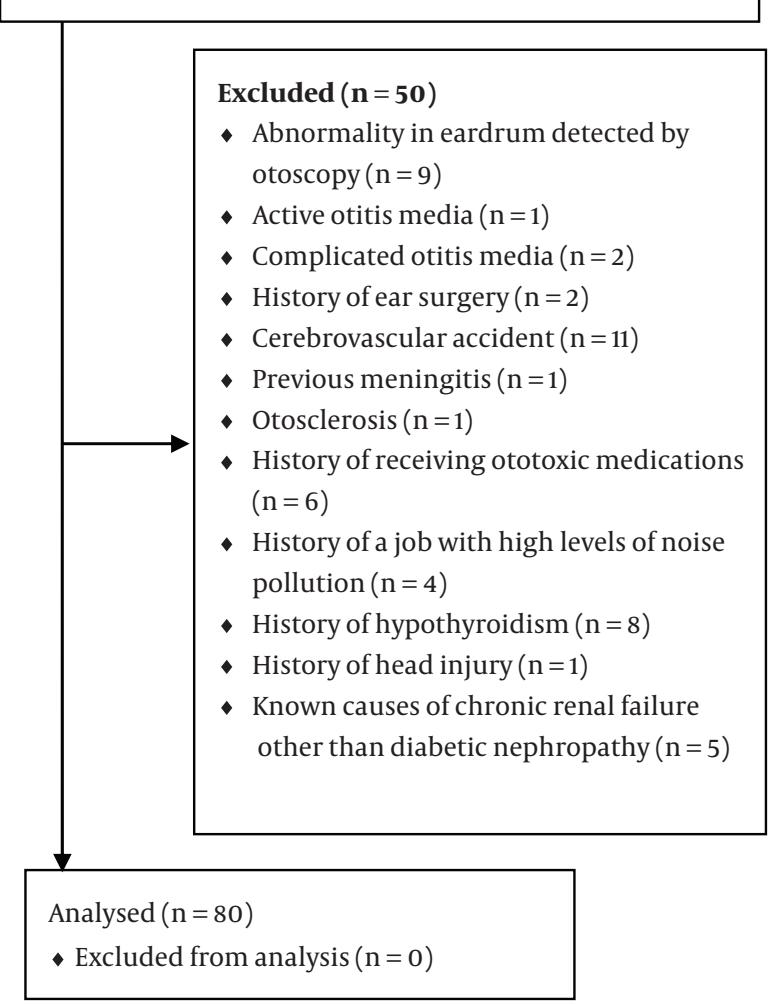

Figure 1. The Number of Excluded Patients and the Cause of Exclusion in Both Groups

\section{Discussion}

The present study showed that hearing loss in high frequency sounds was more common in the diabetics compared with healthy controls. Among the diabetics, an increase in the prevalence of hearing loss was observed with an increase in the sound frequency. The prevalence of hearing loss was increased from $11.2 \%$ at the sound frequency of $250 \mathrm{~Hz}$ to $41.2 \%$ at the frequency of $8 \mathrm{KHz}$. The comparison of the mean hearing threshold in the right ears of the two groups showed that the diabetics and the controls were significantly different in all sound frequencies except for the frequency of $250 \mathrm{~Hz}$. The mean hearing threshold was within the normal range at all the frequencies among the controls. At the same time, the mean hearing threshold in the diabetics was out of the normal range at the frequencies of $1 \mathrm{KHz}$ to $8 \mathrm{KHz}$. This finding signifies the hearing loss at high frequencies among the diabetics. The comparison of the mean hearing threshold of the left ears in the two groups showed that the diabetic and the non-diabetic people were significantly different in all sound frequencies over $1 \mathrm{KHz}$. The mean hearing threshold was within the nor- mal range at all sound frequencies in the controls. Meanwhile, the mean hearing threshold was out of the normal range in the frequencies of $2 \mathrm{KHz}$ and more among the diabetics.

High levels of serum creatinine might not only be a sign of poorly controlled diabetes, but also they are associated with severe hearing loss.

Previous studies have shown that hearing loss is associated with reduced social relationships, depression, cognitive disorders, and the poor quality of life (10-16). Chronic exposure to excessive noise, chemical, ototoxic drugs, cigarette smoke, alcohol, high blood pressure, and a history of head trauma are among the risk factors of hearing impairment (17-22). The review of literature shows controversial results on the relationship between diabetes and hearing impairment. Several studies have shown that hearing loss is more common in the diabetics than in the non-diabetic people $(6,7)$. On the other hand, there are studies that did not find a significant relationship between diabetes and hearing impairment $(8,9)$. The controversies among the results might be due to the inconsistencies in 
Table 1. The Patients' Characteristics in the Two Study Groups ${ }^{\mathrm{a}}$

\begin{tabular}{|c|c|c|c|}
\hline \multirow[t]{2}{*}{ Variable } & \multicolumn{2}{|c|}{ Group } & \multirow[t]{2}{*}{ P Value } \\
\hline & Diabetics & Controls & \\
\hline Age, $y$ & $44.97 \pm 6.21$ & $43.42 \pm 5.80$ & 0.18 \\
\hline Gender & & & 0.07 \\
\hline Male & $35(43.75)$ & $37(46.25)$ & \\
\hline Female & $45(56.25)$ & $43(53.75)$ & \\
\hline BMI, $\mathrm{kg} / \mathrm{m}^{2}$ & & & 0.04 \\
\hline Healthy weight & $31(38.7)$ & $42(52.5)$ & \\
\hline Overweight & $32(40)$ & $34(42.5)$ & \\
\hline Obese & $17(21.3)$ & $4(5)$ & \\
\hline Creatinine, $\mathrm{mg} / \mathrm{dL}$ & $1.01 \pm 0.21$ & $0.99 \pm 0.21$ & 0.56 \\
\hline Glomerular Filtration Rate & $88.82 \pm 23.62$ & $91.22 \pm 24.67$ & 0.61 \\
\hline HbA1c, mg/dL & $9.09 \pm 2.03$ & $3.84 \pm 0.67$ & $<0.001$ \\
\hline
\end{tabular}

Abbreviations: $\mathrm{Kg} / \mathrm{m}^{2}$, kilogram per square meter; $\mathrm{mg} / \mathrm{dL}$, milligram per deciliter

${ }^{\mathrm{a}}$ Values are expressed as mean \pm standard deviation (SD) or No. (\%).

Table 2. The Distribution of Hearing Loss Among the Two Study Groups ${ }^{\mathrm{a}}$

\begin{tabular}{l|c|c|c}
\hline \multirow{2}{*}{ Sounds Intensity } & Hearing status & Diabetic & Controls \\
\hline \multirow{2}{*}{$\mathbf{2 5 0 ~ H z}$} & Normal & $71(88.8)$ & $78(97.5)$ \\
\cline { 2 - 4 } & Hearing loss & $9(11.2)$ & $2(2.5)$ \\
\cline { 2 - 4 } & Normal & $69(86.3)$ & $80(100)$ \\
\hline \multirow{2}{*}{$\mathbf{1 ~ K H z}$} & Hearing loss & $11(13.7)$ & 0 \\
\hline \multirow{2}{*}{$\mathbf{2} \mathbf{~ K H z}$} & Normal & $68(85)$ & $80(100)$ \\
\cline { 2 - 4 } & Hearing loss & $12(15)$ & 0 \\
\hline \multirow{2}{*}{$\mathbf{4} \mathbf{~ K H z}$} & Normal & $65(81.3)$ & $80(100)$ \\
\cline { 2 - 4 } & Hearing loss & $15(18.7)$ & 0 \\
\hline \multirow{2}{*}{$\mathbf{8} \mathbf{~ K H z}$} & Normal & $52(65)$ & $78(97.5)$ \\
\cline { 2 - 4 } & Hearing loss & $28(35)$ & $2(2.5)$ \\
\hline
\end{tabular}

${ }^{\mathrm{a}}$ Values are expressed as No. (\%).

the methods, lack of appropriate matched controls, and the problems in studying the effects of confounders (such as hypertension, atherosclerosis, and glycemic control) on the hearing of the diabetics. Therefore, well designed studies considering the possible confounders in the hearing loss of the diabetics are suggested.

Microangiopathic lesions in the inner ear, auditory nerve neuropathy, and even mutations in the mitochondrial genes have been listed as the possible causes of hear- ing impairment in diabetes (23). The previous research stressed on the need to determine the subtypes of diabetes that are at risk of hearing impairment (24). Considering the results of this study, high levels of creatinine in the diabetic patients might explain a potential mechanism for hearing impairment in these patients. The eardrum lacks blood vessels. Its nutrition and waste material removal is mostly done through diffusion. Therefore, any changes in the osmolarity of body fluids, might directly affect the process of diffusion through the eardrum. Increase in the concentration of the substances with the greatest impact on the osmolarity of the body fluids, may result in an osmotic shock in the eardrum which consequently decreases its elasticity and reduces its ability to conduct sound vibrations.

\subsection{Clinical Implication}

Appropriate audiometric screening is recommended for the diabetics especially in those with increased serum creatinine level. Timely management of the hearing impairment in these high risk patients could result in the improvement of their quality of life.

\subsection{Conclusions}

According to the results of the present study, hearing impairment in the high frequency sounds was more common in the diabetics than healthy individuals. Also, the severity of hearing loss in patients with diabetes was associated with the levels of creatinine (diabetic nephropathy). The hypothesis of the relationship between diabetes and hearing loss through nephropathy should be examined more in future studies.

\section{Acknowledgments}

The authors express their gratitude to the technicians in the audiometry ward of Matini hospital.

\section{Footnotes}

Authors' Contribution: Saeed Nouri and Kurosh Gharagozli: study concept, study design, and data collection; Raika Jamali, Mohammad Reza Sharif, and Bardia Jamali: performing statistical analysis, interpretation of the data, drafting the manuscript, critical revision of the manuscript for important intellectual content, and study supervision.

Financial Disclosure: No benefits in any form have been received or would be received from a commercial party related directly or indirectly to the subject of this article. 
Table 3. The Comparison of Mean Serum Creatinine Level in the Diabetic Patients with and Without Hearing Loss

\begin{tabular}{|c|c|c|c|c|}
\hline Sounds Intensity & Hearing Status & Number & Creatinine $^{a}$ & P Value \\
\hline \multirow{2}{*}{$250 \mathrm{~Hz}$} & Normal & 71 & $1.02 \pm 0.21$ & 0.63 \\
\hline & Hearing loss & 9 & $0.97 \pm 0.24$ & \\
\hline \multirow{2}{*}{$500 \mathrm{~Hz}$} & Normal & 69 & $1.0 \pm 0.20$ & 0.22 \\
\hline & Hearing loss & 11 & $1.19 \pm 0.26$ & \\
\hline \multirow{2}{*}{$1 \mathbf{K H z}$} & Normal & 68 & $0.99 \pm 0.19$ & 0.09 \\
\hline & Hearing loss & 12 & $1.14 \pm 0.27$ & \\
\hline \multirow{2}{*}{$2 \mathbf{K H z}$} & Normal & 65 & $0.98 \pm 0.19$ & 0.05 \\
\hline & Hearing loss & 15 & $1.13 \pm 0.26$ & \\
\hline \multirow{2}{*}{$4 \mathrm{KHz}$} & Normal & 52 & $0.96 \pm 0.17$ & 0.01 \\
\hline & Hearing loss & 28 & $1.11 \pm 0.26$ & \\
\hline \multirow{2}{*}{$8 \mathrm{KHz}$} & Normal & 47 & $0.95 \pm 0.17$ & 0.005 \\
\hline & Hearing loss & 33 & $1.10 \pm 0.24$ & \\
\hline
\end{tabular}

${ }^{\mathrm{a}}$ Values are expressed as mean \pm standard deviation (SD).

Funding/Support: This study was conducted by the financial support of vice chancellor for research at Kashan University of Medical Sciences (grant No: 9155).

\section{References}

1. Virtaniemi J, Laakso M, Nuutinen J, Karjalainen S, Vartiainen E. Hearing thresholds in insulin-dependent diabetic patients. J Laryngol Otol. 1994;108(10):837-41. [PubMed: 7989828].

2. Zimmet PZ, McCarty DJ, de Courten MP. The global epidemiology of non-insulin-dependent diabetes mellitus and the metabolic syndrome. J Diabetes Complicat. 1997;11(2):60-8. doi: 10.1016/s10568727(96)00090-6.

3. Rajendran S, Anandhalakshmi MB, Vishwanathan R. Evaluation of the incidence of sensorineural hearing loss in patients with type 2 diabetes mellitus. Int J Biol Med Res. 2011;2(4):982-7.

4. Vaughan N, James K, McDermott D, Griest S, Fausti S. A 5-year prospective study of diabetes and hearing loss in a veteran population. Otol Neurotol. 2006;27(1):37-43. [PubMed: 16371845].

5. Qaiyum HA, Tamkanth A, Siraj M, Parveen N, Ishaq M. A study on the incidence of Sensorineural hearing loss in patients with Diabetes mellitus. Int J. 2015;3(2):685-7.

6. Malucelli DA, Malucelli FJ, Fonseca VR, Zeigeboim B, Ribas A, Trotta F, et al. Hearing loss prevalence in patients with diabetes mellitus type 1. Braz J Otorhinolaryngol. 2012;78(3):105-15. [PubMed: 22714855].

7. Taziki Mohammad H, Mansourian Azad R. The comparison of hearing loss among diabetic and non-diabetic patients.J Clin Diagnos Res. 2011;5(1):88-90.

8. Kurien M, Thomas K, Bhanu TS. Hearing threshold in patients with diabetes mellitus. J Laryngol Otol. 1989;103(2):164-8. [PubMed: 2926261].

9. Sieger A, White NH, Skinner MW, Spector GJ. Auditory function in children with diabetes mellitus. Ann Otol Rhinol Laryngol. 1983;92(3 Pt 1):237-41. [PubMed: 6344724].

10. Herbst KG, Humphrey C. Hearing impairment and mental state in the elderly living at home. Br Med J. 1980;281(6245):903-5. [PubMed: 7427503].

11. Dalton DS, Cruickshanks KJ, Klein BE, Klein R, Wiley TL, Nondahl DM. The impact of hearing loss on quality of life in older adults. Gerontologist. 2003;43(5):661-8. [PubMed: 14570962].
12. Gates GA, Cobb JL, Linn RT, Rees T, Wolf PA, D’Agostino RB. Central auditory dysfunction, cognitive dysfunction, and dementia in older people. Arch Otolaryngol Head Neck Surg. 1996;122(2):161-7. [PubMed: 8630210].

13. Jankar DS, Bodhe CD, Bhutada TB. A study on hearing loss in type II diabetics. Int J Med Res Health Sci. 2013;2(4):893. doi: 10.5958/j.23195886.2.4.143.

14. Ebert DA, Heckerling PS. Communication with deaf patients. Knowledge, beliefs, and practices of physicians. JAMA. 1995;273(3):227-9. [PubMed: 7807662]

15. Gates GA, Cobb JL, D'Agostino RB, Wolf PA. The relation of hearing in the elderly to the presence of cardiovascular disease and cardiovascular risk factors. Arch Otolaryngol Head Neck Surg. 1993;119(2):156-61. [PubMed: 8427676].

16. Barnett S, Franks P. Deafness and mortality: analyses of linked data from the National Health Interview Survey and National Death Index. Public Health Rep. 1999;114(4):330-6. [PubMed:10501133].

17. Mulroy MJ, Henry WR, McNeil PL. Noise-induced transient microlesions in the cell membranes of auditory hair cells. Hear Res. 1998;115(12):93-100. [PubMed: 9472738].

18. Johnson AC, Nylen PR. Effects of industrial solvents on hearing. Occup Med. 1995;10(3):623-40. [PubMed: 8578423].

19. Fitzgerald DC. Head trauma: hearing loss and dizziness. J Trauma. 1996;40(3):488-96. [PubMed: 8601878].

20. Aran JM, Hiel H, Hayashida T. In: Noise Induced Hearing Loss. Dancer A, Henderson D, Salvi R, Hamernik R, editors. 5. St Louis: Mosby; 1992. pp.188-95.Noise, aminoglycosides, diuretics.

21. Rosenhall U, Sixt E, Sundh V, Svanborg A. Correlations between presbyacusis and extrinsic noxious factors. Audiology. 1993;32(4):234-43. [PubMed: 8343080].

22. Brant LJ, Gordon-Salant S, Pearson JD, Klein LL, Morrell CH, Metter EJ, et al. Risk factors related to age-associated hearing loss in the speech frequencies. J Am Acad Audiol. 1996;7(3):152-60. [PubMed: 8780987].

23. Janssen GM, Maassen JA, van Den Ouweland JM. The diabetesassociated 3243 mutation in the mitochondrial tRNA(Leu(UUR)) gene causes severe mitochondrial dysfunction without a strong decrease in protein synthesis rate. J Biol Chem. 1999;274(42):29744-8. [PubMed: 10514449]. 
24. Fukui M, Kitagawa Y, Nakamura N, Kadono M, Mogami S, Ohnishi M, et al. Idiopathic sudden hearing loss in patients with type 2 diabetes.
Diabetes Res Clin Pract. 2004;63(3):205-11. [PubMed:14757292]. 